\title{
Recent Developments in Artificial Intelligence-Based Techniques for Prostate Cancer Detection: A Scoping Review
}

\author{
Uzair SHAH ${ }^{\mathrm{a}}$, Md. Rafuil BISWAS ${ }^{\mathrm{a}}$, Mahmood Saleh ALZUBAIDI ${ }^{\mathrm{a}}$, Hazrat ALI ${ }^{\mathrm{a}}$, \\ Tanvir ALAM ${ }^{\mathrm{a}}$, Mowafa HOUSEH ${ }^{\mathrm{a}}$ and Zubair SHAH ${ }^{\mathrm{a}, 1}$ \\ ${ }^{a}$ College of Science and Engineering, Hamad Bin Khalifa University, Qatar \\ Foundation, Doha, Qatar
}

\begin{abstract}
Artificial intelligence (AI) techniques can contribute to the early diagnosis of prostate cancer. Recently, there has been a sharp increase in the literature on AI techniques for prostate cancer diagnosis. This review article presents a summary of the AI methods that detect and diagnose prostate cancer using different medical imaging modalities. Following the PRISMA-ScR principle, this review covers 69 studies selected from 1441 searched papers published in the last three years. The application of AI methods reported in these articles can be divided into three broad categories: diagnosis, grading, and segmentation of tissues that have prostate cancer. Most of the AI methods leveraged convolutional neural networks (CNNs) due to their ability to extract complex features. Some studies also reported traditional machine learning methods, such as support vector machines (SVM), decision trees for classification, LASSO, and Ridge regression methods for features extraction. We believe that the implementation of AI-based tools will support clinicians to provide better diagnosis plans for prostate cancer.
\end{abstract}

Keywords. Prostate cancer, medical imaging, machine learning, deep learning.

\section{Introduction}

After lung cancer, prostate cancer is the second most common cancer in men [1]. Reports have projected that the number of prostate cancer cases may exceed the number of lung cancer cases in men in just over a decade [1], [2]. However, early diagnosis of prostate cancer can decrease the fatality and morbidity rates. In clinical practice, the diagnosis is typically performed by a transrectal ultrasound and blood tests for prostatespecific antigens [3]. Usually, the severity of prostate cancer is measured in terms of Gleason score (ranked from 6 to 10), with a higher score representing high-grade cancer that is more likely to spread in the tissue [4], [5]. Analyzing and grading prostate cancer scores require trained professionals, who usually rank the scores through manual screening and mutual consensus of many experts relying on their skills.

The AI research community has made progress in developing AI-based methods to support pathologists and radiologists, thus improving the overall efficiency of the process of diagnosing prostate cancer [6]. Typically, AI-based methods can enable quick

${ }^{1}$ Corresponding Author. Zubair Shah, College of Science and Engineering, Hamad Bin Khalifa University, Qatar Foundation, Doha, Qatar. Email: zshah@hbku.edu.qa 
processing and accelerate the diagnosis process while ensuring consistency [7]. In prostate cancer, AI assists in systematic pathological grading to evaluate prostate cancer stratification and care [8]. While many published methods exist proposing the use of AI to diagnose prostate cancer, there are few comprehensive review that may provide a quick insight to readers exploring the recent developments in AI's use for studying prostate cancer. This short review will serve as a quick reference for readers interested in studying and researching the role of AI methods in treating prostate cancer.

\section{Methods}

We performed a scoping review to highlight the advancements of AI-based tools detecting prostate cancer. We followed the guidelines from the Preferred Reporting Items for Systematic Review and Meta-Analysis (PRISMA-ScR) [9] to perform the review (Appendix 1). The search strategy, which included population (i.e., prostate cancer), target intervention (i.e., artificial intelligence), and target outcome (i.e., diagnosis), was applied to the most widely used databases (PubMed, Medline, Embase, and Google Scholar) in the medical domain. The search process was carried between February 10th and $14^{\text {th }}, 2021$ and extracted relevant articles published over the last three years covering the most recent publications in this domain.

\section{Results and Discussion}

A total of 1476 studies were retrieved by searching four databases (details in Appendix 4). Amongst these, 574 duplicate studies were excluded. Through title and abstract screening, 767 studies were excluded following the exclusion criterion of being non-English studies, non-peer-reviewed articles, or scoping review text. Through fulltext screening of the remaining 135 studies, 66 studies were further removed following the exclusion criteria. A total of 69 studies were included in this review. The key characteristics of the articles surveyed are summarized in Table 1.

Table 1: Characteristics of AI-based techniques and data modalities reported for prostate cancer.

\begin{tabular}{|c|c|c|c|c|c|}
\hline Characteristics & \multicolumn{5}{|c|}{ Number of Studies } \\
\hline Model purpose & \multicolumn{2}{|c|}{ Diagnosis of prostate: 43} & \multicolumn{2}{|c|}{ Grading prostate: 15} & Segmentation:11 \\
\hline AI Branches & \multicolumn{2}{|c|}{ Deep Learning: 49} & \multicolumn{2}{|c|}{$\begin{array}{l}\text { Machine Learning } \\
\text { and Deep Learning: } 2\end{array}$} & $\begin{array}{l}\text { Segmentation:11 } \\
\text { Machine } \\
\text { Learning:18 }\end{array}$ \\
\hline AI method & $\mathrm{CNN}: 45$ & ANN:4 & SVM:4 & $\begin{array}{l}\text { Random } \\
\text { Forest: } 3\end{array}$ & $\begin{array}{l}\text { Logistic } \\
\text { Regression: } 2\end{array}$ \\
\hline $\begin{array}{l}\text { Feature } \\
\text { Extraction } \\
\text { Technique }\end{array}$ & $\begin{array}{l}\text { CNN:45 } \\
\text { LASSO: } 2 \\
\text { Radiomics } \\
: 4\end{array}$ & $\begin{array}{l}\text { LB-FCN light:1 } \\
\text { CSLBP: } 1 \\
\text { CFS: } 1\end{array}$ & $\begin{array}{l}\text { DCE: } 1 \\
\text { SFIT \& } \\
\text { EFDs: } 1 \\
\text { CDF \& } \\
\text { ADC: } 1\end{array}$ & $\begin{array}{l}\text { Genetic } \\
\text { Algorithm:1 } \\
\text { FCC:1 } \\
\text { Mean Region } \\
\text { of Interest:1 }\end{array}$ & $\begin{array}{l}\text { Histogram:1 } \\
\text { Gray level co- } \\
\text { occurrence } \\
\text { matrix:1 } \\
\text { NA:8 }\end{array}$ \\
\hline $\begin{array}{l}\text { Imaging } \\
\text { modality }\end{array}$ & $\begin{array}{l}\text { MRI/PET/ } \\
\text { CT: } 34\end{array}$ & $\begin{array}{l}\text { Biopsy whole } \\
\text { slide images: } 13\end{array}$ & $\begin{array}{l}\text { Tissue Micro } \\
\text { arrays: } 6\end{array}$ & $\begin{array}{l}\text { Histopatholog } \\
\text { ical data: } 04\end{array}$ & Genetic Data: 03 \\
\hline \multicolumn{6}{|c|}{$\begin{array}{l}\text { Index: ANN: Artificial Neural Network. CSLBP: completed and statistical local binary pattern. CFS: } \\
\text { Correlation Feature Selection. DCE: Dynamic contrast-enhanced. SFIT: Scale-invariant feature transforms. } \\
\text { EFDs: Elliptic Fourier Descriptors. CDF: Cumulative Distribution function. ADC: Apparent Diffusion } \\
\text { Coefficient. FCC: Frequency Cepstral Coefficients. MRI: Magnetic Resonance Imaging. PET: Positron } \\
\text { Emission Tomography. CT: Computed Tomography }\end{array}$} \\
\hline
\end{tabular}


Out of these 69 studies, $94 \%(n=65)$ were peer-reviewed journal articles, while the remaining were conference papers. Of these, $20 \%(n=12)$ were published in 2018 , $39 \%(n=26)$ in 2019 , and $36 \%(n=25)$ in 2020 . The highest number of studies were published from the United States $(n=16)$, followed by China $(n=11)$ and Canada $(n=8)$ (Appendix 2).

For the majority of the studies, the data was acquired from public repositories such as the ProstateX challenge dataset, PRMISE-12 dataset, and NCBI. The most dominant imaging modalities were magnetic resonance imaging (MRI), positron emission tomography (PET) scan, and computerized tomography (CT) scan. The datasets' descriptions are summarized in Table 2.

Table 2: Descriptions and Statistics of Public and Private Dataset for Prostate Cancer

\begin{tabular}{|c|c|c|c|c|}
\hline Dataset & Host/Source & Type of data & $\begin{array}{l}\text { Size (No. } \\
\text { of } \\
\text { samples) }\end{array}$ & $\begin{array}{l}\text { Studies that reported } \\
\text { the use (listed in } \\
\text { Appendix 3) }\end{array}$ \\
\hline \multirow{10}{*}{$\begin{array}{l}\text { Public } \\
\text { Dataset }\end{array}$} & clinicaltrials.gov $(\mathrm{NIH})$ & PET/MRI & 122 & 42 \\
\hline & $\begin{array}{l}\text { Registry of Catastrophic } \\
\text { Illness Patient (subpart NIH) }\end{array}$ & $\begin{array}{l}\text { Electronic Health } \\
\text { Record (EHR) }\end{array}$ & 20355 & 62 \\
\hline & $\begin{array}{l}\text { Harvard Medical School and } \\
\text { Brigham woman's hospital }\end{array}$ & MRI & 682 & 52 \\
\hline & GLOBOCAN 2018 & Ultrasound & 1200 & 48 \\
\hline & Prostate $\mathrm{X}$ challenge data & mp-MRI & 538 & $50,20,38$ \\
\hline & $\begin{array}{ll}\text { National Centre } & \text { of } \\
\text { Biotechnology (NCBI) } & \end{array}$ & Genetic dataset & 179 & 25,67 \\
\hline & $\begin{array}{lr}\text { MICCAI Prostate MR Image } \\
\text { Segmentation } \\
\text { (PROMISE12) }\end{array}$ & MRI & 4050 & 32,70 \\
\hline & ProstateGlandDB dataset & $\begin{array}{l}\text { Biopsy whole } \\
\text { slides images }\end{array}$ & 35 & 13,64 \\
\hline & Horosproject.org & PET Images & 7336 & 71 \\
\hline & NCI-ISBI 2013 challenge & MRI & 771 & 5 \\
\hline \multirow{3}{*}{$\begin{array}{l}\text { Private } \\
\text { Datasets }\end{array}$} & Boramae Medical Center & EHR & 3791 & 40 \\
\hline & $\begin{array}{l}\text { The University of Alabama } \\
\text { at Birmingham (UAB) }\end{array}$ & MRI Scan & 1269 & 22 \\
\hline & $\begin{array}{l}\text { Univ. of Texas Southwestern } \\
\text { Medical Center }\end{array}$ & CT scan & 85 & 19 \\
\hline
\end{tabular}

Features extraction techniques were reported in 58 studies. Most of the included studies used CNN ( $\mathrm{n}=45$, listed as 3, 5, 6, 7, 9, $10-12,14,15,18,20-22,24,25,28$, $33-37,39,42,43,45-49,50,53,55,57,58,60-64,66-70$ in Appendix 3) followed by radiomics ( $\mathrm{n}=4$, listed as 30, 31, 38, 41 in Appendix 3) and LASSO ( $\mathrm{n}=2$, listed as 4, 44 in Appendix 3). Other reported methods for feature extractions were gray level cooccurrence matrix (54 in Appendix 3), mean region of interest (71 in Appendix 3), genetic algorithms (13 in Appendix 3), completed and statistical local binary pattern (52 in Appendix 3), apparent diffusion coefficient and cumulative distribution function (19 in Appendix 3), frequency cepstral coefficient (26 in Appendix 3), and correlation features selection algorithm (32 in Appendix 3).

It is challenging to compare the performance of the studies as each study utilized different feature extraction techniques, imaging modility and performance metrics. However, CNN were the prominent feature extraction techniques regardless of the imaging modility [10]. This may be in part because $\mathrm{CNN}$ is sensitive to the training data compared to radiomics and usually requires large data for better performance. 


\section{Conclusion and Future Direction}

This review article identifies three main themes on prostate cancer detection, i.e., diagnosis, grading, and segmentation of histopathological images where AI-based methods were leveraged. We could not find significant application of the AI-based methods for the treatment and recommended medications for prostate cancer. The studies were categorized based on the usage of the AI methods, feature extraction techniques, and types of the dataset used. The use of these AI techniques are limited to academic and research purposes only and their real-life applications into clinical practice are currently limited - though a few cases are available where the AI-based tools have been used in clinical practices. Nevertheless, with the rapid progress of AI, technology readiness levels need to be improved for utilizing these methods in real-life diagnosis of prostate cancer.

The appendix is available at https://github.com/hazratali/appendix

\section{References}

[1] Sung H, Ferlay J, Siegel RL, Laversanne M, Soerjomataram I, Jemal A, Bray F. Global Cancer Statistics 2020: GLOBOCAN Estimates of Incidence and Mortality Worldwide for 36 Cancers in 185 Countries. CA Cancer J Clin. 2021 May;71(3):209-249. doi: 10.3322/caac.21660. Epub 2021 Feb 4. PMID: 33538338

[2] Report by World Cancer Research Foundation, available at: https://www.wcrf.org/dietandcancer/prostate-cancer/ (Accessed: 25 August 2021)

[3] Aldoj N, Lukas S, Dewey M, Penzkofer T., "Semi-automatic classification of prostate cancer on multiparametric MR imaging using a multi-channel 3D convolutional neural network," Eur. Radiol., vol. 30, no. 2, pp. 1243-1253, 2020, doi: 10.1007/s00330-019-06417-z

[4] H. Bonkhoff, "Gleason grading: diagnostic criteria and clinical implications.," Pathologe, vol. 26, no. 6, pp. 422-432, Nov. 2005, doi: 10.1007/s00292-005-0793-y

[5] Reda, Islam, Babajide O. Ayinde, Mohammed Elmogy, Ahmed Shalaby, Moumen El-Melegy, Mohamed Abou El-Ghar, Ahmed Abou El-fetouh, Mohammed Ghazal, and Ayman El-Baz. "A new CNN-based system for early diagnosis of prostate cancer." In 2018 IEEE 15 th International Symposium on Biomedical Imaging (ISBI 2018), pp. 207-210. IEEE, 2018. doi: 10.1109/ISBI.2018.8363556

[6] Pantanowitz, Liron, Gabriela M. Quiroga-Garza, Lilach Bien, Ronen Heled, Daphna Laifenfeld, Chaim Linhart, Judith Sandbank et al. "An artificial intelligence algorithm for prostate cancer diagnosis in whole slide images of core needle biopsies: a blinded clinical validation and deployment study." Lancet Digit. Heal., vol. 2, no. 8, pp. e407-e416, 2020, doi: 10.1016/S2589-7500(20)30159-X

[7] Egevad, Lars, Daniela Swanberg, Brett Delahunt, Peter Ström, Kimmo Kartasalo, Henrik Olsson, Dan M. Berney et al. "Identification of areas of grading difficulties in prostate cancer and comparison with artificial intelligence assisted grading." Virchows Arch., vol. 477, no. 6, pp. 777-786, 2020, doi: 10.1007/s00428-020-02858-w

[8] S. A. Harmon, S. Tuncer, T. Sanford, P. L. Choyke, and B. Türkbey, "Artificial intelligence at the intersection of pathology and radiology in prostate cancer.," Diagn. Interv. Radiol., vol. 25, no. 3, pp. 183-188, May 2019, doi: 10.5152/dir.2019.19125

[9] Tricco, A.C., Lillie, E., Zarin, W., O'Brien, K.K., Colquhoun, H., Levac, D., Moher, D., Peters, M.D., Horsley, T., Weeks, L. and Hempel, S., "PRISMA extension for scoping reviews (PRISMA-ScR): Checklist and explanation,” Annals of Internal Medicine, vol. 169, no. 7. pp. 467-473, Oct. 02, 2018, doi: 10.7326/M18-0850

[10] Daniel Truhn, Simone Schrading, Christoph Haarburger, Hannah Schneider, Dorit Merhof, and Christiane Kuhl "Radiomic versus Convolutional Neural Networks Analysis for Classification of Contrast enhancing Lesions at Multiparametric Breast MRI” Radiology 2019 290:2, 290-297, doi: 10.1148/radiol.2018181352 